\title{
Ratio of proliferation markers and HSP90 gene expression as a predictor of pathological complete response in breast cancer neoadjuvant chemotherapy
}

\author{
Michal Jarzab', Monika Kowal'², Wieslaw Bal'1, Malgorzata Oczko-Wojciechowska², \\ Justyna Rembak-Szynkiewicz ${ }^{3}$, Malgorzata Kowalska², Ewa Stobiecka ${ }^{4}$, Ewa Chmielik', \\ Tomasz Tyszkiewicz ${ }^{2}$, Malgorzata Kaszuba ${ }^{3}$, Elzbieta Nowicka ${ }^{1}$, Barbara Lange ${ }^{1}$, \\ Agnieszka Czarniecka ${ }^{5}$, Jolanta Krajewska ${ }^{2}$, Alicja Dyla ${ }^{3}$, Miroslaw Dobrut ${ }^{5}$, \\ Dariusz Lange $^{4}$, Barbara Jarzab², Barbara Bobek-Billewicz ${ }^{3}$, Rafal Tarnawski ${ }^{1}$
}

${ }^{1} 3^{\text {rd }}$ Department of Radiotherapy and Chemotherapy, Breast Cancer Center, Maria Sklodowska-Curie Memorial Cancer Center and Institute of Oncology, Gliwice Branch, Gliwice, Poland

${ }^{2}$ Laboratory of Molecular Diagnostics and Functional Genomics, Department of Nuclear Medicine and Endocrine Oncology, Maria Sklodowska-Curie Memorial Cancer Center and Institute of Oncology, Gliwice Branch, Gliwice, Poland

${ }^{3}$ Department of Radiology, Maria Sklodowska-Curie Memorial Cancer Center and Institute of Oncology, Gliwice Branch, Gliwice, Poland

${ }^{4}$ Department of Tumor Pathology, Maria Sklodowska-Curie Memorial Cancer Center and Institute of Oncology, Gliwice Branch, Gliwice, Poland

${ }^{5}$ Department of Oncological Surgery, Maria Sklodowska-Curie Memorial Cancer Center and Institute of Oncology, Gliwice Branch, Gliwice, Poland

\begin{abstract}
Introduction. Prediction of response to preoperative breast cancer chemotherapy may offer a substantial optimization of medical management of this disease. The most efficient prediction would be done $a$ priori, before the start of chemotherapy and based on the biological features of patient and tumor. Numerous markers have been proposed but none of them has been applied as a routine. The role of MKI67 and HSP90 expression has been recently suggested to predict treatment sensitivity in HER2-positive breast cancer. The aim of this study was to validate the utility of proliferation based markers (MKI67 and CDK1) and heat shock proteins (namely HSP90) to predict response to chemotherapy in cohort of breast cancer patients treated preoperatively.

Material and methods. Ninety-three patients with breast cancer, all females, mean age 42.2 years, among them $32 \%$ T1-T2 patients, $49 \%$ T3 patients and 13\% with T4 tumor stage, $27 \%$ N0, $42 \%$ N1, 16\% N2, $15 \%$ N3 were subjected to initial chemotherapy. The majority of patients $(86 \%)$ received anthracycline and taxane chemotherapy. Among the patients there were 9 individuals with metastatic disease (M1) at initial presentation, and 11 patients were not treated surgically after initial chemotherapy (no sufficient disease response). From 82 patients operated on, 20 patients $(24 \%)$ showed pathological complete response (pCR), while in 62 patients there was
\end{abstract}

Correspondence address: M. Jarzab, M.D., Ph.D.

$3^{\text {rd }}$ Dept. of Radiotherapy and Chemotherapy, Breast Cancer Unit

Maria Sklodowska-Curie Memorial Cancer Center

and Institute of Oncology, Gliwice Branch

Wybrzeze Armii Krajowej 15, 44-101 Gliwice, Poland

tel.: +4832278 86 17, fax: +4832278 8441

e-mail: michal.jarzab@io.gliwice.pl 
no pCR. $42 \%$ of patients were hormone-sensitive HER2-negative, 20\% hormone-sensitive HER2-positive, 9\% only HER-positive and $29 \%$ with triple negative breast cancer.

Four gene transcripts (MKI67, cyclin-dependent kinase 1 [CDK1], heat shock proteins HSP90AA1 and HSP90AB1) were analyzed in total RNA isolated from single core obtained during preoperative core needle biopsy by quantitative real-time PCR with fluorescent probes (Universal Probe Library, Roche). Results were normalized to the panel of reference genes.

Results. There were no statistically significant differences in MKI67 and CDK1 expression between pCR and no pCR groups ( $p=0.099$ and 0.35 , respectively), although the median expression of both genes was slightly higher in pCR group. In contrast, both HSP90AA1 and HSP90AB1 transcripts showed decreased expression in pCR group (medians 0.77 and 0.55 ) when compared to no pCR group (median 0.86 and 0.73 ), statistically significant for HSP90AA1 $(p=0.031)$ and of borderline significance for HSP90AB1 $(p=0.054)$.

The most significant predictor of $\mathrm{pCR}$ was the ratio of CDK1 transcript to HSP90AA transcript. This ratio was significantly higher in CR group (median 0.99 ) than in no CR group (median 0.68, p =0.0023), and showed a potential diagnostic utility (area under receiver operating characteristic [ROC] curve 0.72 ).

Conclusions. HSP90AA1 and AB1 genes exhibit low expression in breast cancers highly sensitive to chemotherapy and may indicate the patients with higher probability of pathological complete response. The ratio of HSP90AA1 to proliferation-related markers (CDK1 or MKI67) may be even better predictor of pCR chance, with higher expression of proliferation genes and lower stress response in patients sensitive to chemotherapy. (Folia Histochemica et Cytobiologica 2016, Vol. 54, No. 4, 202-209)

Key words: breast cancer; chemotherapy; chemosensitivity; pathological complete response; proliferation markers; HSP90AA1; HSP90AB1; gene expression

\section{Introduction}

Prediction of response to preoperative breast cancer chemotherapy may offer a substantial optimization of medical management of this disease. The most efficient prediction would be done a priori, before the start of chemotherapy and would be based on the biological features of patient and tumor. This approach allows for the selection of patients who may benefit from therapy and may aid the choice of treatment regimen. The alternative approach is the prediction made after the therapy has been initiated, either based on the treatment response visualization by imaging studies or by the change of biomarkers representing the disease burden (the latter currently less developed, e.g. serum markers).

The idea of a priori prediction has been evaluated in many studies; however, no efficient markers have been found and the research slowed down after the broad acceptance of heterogeneity of breast cancer molecular subtypes. These subtypes are applied as a surrogate tool to select patients with chemosensitive disease. Although the subtypes show clearly different biological features, cytotoxic chemotherapy remains the mainstay of treatment in luminal B, HER2-positive and triple negative breast cancer. As novel drugs appear (often less toxic than cytostatics), the importance of selection of patients who may benefit from chemotherapy is growing.

There are many reports suggesting the importance of markers of proliferation, especially the most clinically widespread assessment of MKI67 gene expression, for the chemotherapy response (e.g. Nishimura et al. [1]). Recently, the role of this proliferation-related protein in context of the expression of (among others) heat shock protein HSP90 has been suggested by Bria et al. [2] to predict treatment sensitivity in HER2-positive breast cancer, when chemotherapy was combined with targeted anti-HER 2 treatment. This focused our attention, as previously these two important features of cancer cell (proliferation and shock response) were not related to each other in the context of chemotherapy response prediction in breast cancer. Thus, we undertook the study aiming to assess the utility of proliferation based markers (MKI67 and CDK1) and heat shock proteins (namely HSP90) to predict the response to breast cancer preoperative chemotherapy.

\section{Material and methods}

Ninety-three patients with breast cancer, all females, mean age 42.2 years (95\% confidence interval $40.5-43.9$ years, median 41 years) were enrolled to the study. Patients were selected before the preoperative chemotherapy, either due to locally advanced or oligometastatic breast cancer or early-stage disease with intent of breast-conserving surgery after neoadjuvant chemotherapy. Within the group there were $32 \%$ patients with tumor size T1-T2, $49 \%$ of T3 patients and $13 \%$ females with advanced T4 tumor. No nodal metastases were found in $27 \%$ of patients (cN0), $42 \%$ of patients had N1 nodal involvement, $16 \%$ fixed/matted N2 lymph node metastases and $15 \%$ advanced (N3) lymph node 
involvement. Patients were subjected to initial chemotherapy - the majority of patients $(86 \%)$ received anthracycline and taxane chemotherapy (56\% received docetaxel/ /doxorubicin or epirubicin/cyclophosphamide - TAC/TEC regimen, 19\% doxorubicin/docetaxel AT chemotherapy, $11 \%$ sequential doxorubicin/cyclophosphamide-paclitaxel AC-P regimen), and $14 \%$ anthracycline-based regimen (5-fluorouracil/doxorubicin/cyclophosphamide, FAC). 42\% of patients were hormone-sensitive and HER2-negative, 20\% hormone-sensitive HER2-positive, 9\% non-luminal HER-positive and $29 \%$ with triple negative breast cancer. In total, $64 \%$ of the whole group showed hormone responsiveness (positive estrogen or progesterone staining on immunohistochemical staining, at least $1 \%$ ) and in $29 \%$ of patients positive HER2 status was confirmed (either +++ in immunohistochemical staining or positive FISH in patients with ++ status).

Among the patients there were 9 individuals with metastatic disease (M1) at initial presentation, and 11 patients were not treated surgically after initial chemotherapy (no sufficient disease response). 82 patients reached surgery, and the pathological complete response on postoperative examination was defined as the disappearance of invasive tumor both from tumor bed in breast and axillary lymph nodes. From 82 patients operated on, 20 patients (24\%) showed pathological complete response (pCR), while in 62 patients there was no pCR.

From all patients during pre-treatment core needle biopsy, upon the acceptance of Local Ethics Committee and after the patients' informed consent the additional tissue material was collected and stored in RNAlater. RNA was isolated using RNeasy Mini kits including a digestion step with RNase-free DNase I (Qiagen), its quantity was measured spectrophotometrically (NanoDrop 1000 spectrophotometer, Thermo Scientific), while the quality assessed by Agilent 2100 Bioanalyzer and RNA 6000 Nano LabChip kit (Agilent Technologies).

Four gene transcripts (MKI67, cyclin-dependent kinase 1 [CDK1], heat shock proteins HSP90AA1 and HSP90AB1) were analyzed by quantitative real-time RT-PCR (QPCR) with fluorescent probes, by previously described methodology [3]. Amplicon design was achieved with Universal Probe Library (Roche). Reverse transcription was performed using Qiagen Omniscript RT Kit. The input RNA for each sample was $200 \mathrm{ng}$ in a final volume of $20 \mu \mathrm{L}$. All samples were diluted 10 times prior to QPCR. Reactions were performed in duplicates on 384-well plates using 7900HT Fast Real-Time PCR system (Life Technologies). The QPCR conditions consisted of a first step at $50^{\circ} \mathrm{C}(2 \mathrm{~min}$; activation and incubation with AmpErase UNG) and $95^{\circ} \mathrm{C}$ (10 min; activation AmpliTaq Gold polymerase), followed by 40 cycles of amplification $\left(95^{\circ} \mathrm{C}, 15 \mathrm{~s} ; 60^{\circ} \mathrm{C}, 1 \mathrm{~min}\right)$. Each reaction contained $10 \mu \mathrm{L}$ of Taqman Universal Mastermix (Life Technologies), $200 \mathrm{nM}$ of each primer, $2.8 \mu \mathrm{L}$ RNase- free water and $5 \mu \mathrm{L}$ of diluted cDNA template. Standard curve was performed for each amplicon of the 8 duplicate concentrations (1000 ng, $500 \mathrm{ng}, 200 \mathrm{ng}, 100 \mathrm{ng}, 40 \mathrm{ng}, 20 \mathrm{ng}$, $8 \mathrm{ng}$, and $2 \mathrm{ng}$ of total RNA). The linear regression slope of the standard curve indicated amplification efficiency. For normalization of QPCR data five transcripts were chosen as reference: ACTB, GAPDH, GUSB, PRPLO, TFRC and the normalization factor was obtained using the geNorm applet for Microsoft Excel, based on the three reference genes tested.

\section{Results}

\section{Correlation of analyzed markers}

Four genes were analyzed in the study - MKI67, well-established proliferation marker in breast cancer, cell-cycle related cyclin-dependent kinase 1 , CDK1, and two HSP90 transcripts, HSP90AA1 and HSP90AB1. We assessed the pairwise correlations of four analyzed genes (see Figure 1). There was only a very weak correlation of MKI67 with CDK1 $\left(\mathrm{R}^{2}=\right.$ 0.042). MKI67 was not correlated with two investigated HSP90 transcripts. Instead, there was a moderate correlation between CDK1 and HSP90AA1 and $\mathrm{AB} 1$ transcripts $\left(\mathrm{R}^{2}=0.37\right.$ and 0.35$)$ and moderate correlation between both analyzed HSP90 transcripts $\left(\mathrm{R}^{2}=0.29\right)$.

\section{Gene expression in relation to $p C R$ status}

For MKI67 transcript, mean expression in CR group was 0.74 , in no CR group 0.65 , ratio CR/no CR 1.14 . Median values were 0.72 and 0.54 , respectively, foldchange CR/no CR 1.33 (see Figure 2A). The slight difference between $\mathrm{CR}$ and no $\mathrm{CR}$ groups was not significant ( $\mathrm{p}=0.099$, U Mann-Whitney test).

For CDK1 transcript, there was almost no difference between CR and no CR groups (mean values 0.75 and 0.72 , medians 0.67 and 0.55 , respectively, $p=0.35$, U Mann-Whitney test, Figure 2B).

HSP90AA1 expression was significantly lower in patients with $\mathrm{CR}$ (mean: $0.76,95 \%$ conf. interval $0.59-0.93$, median value 0.77 ) than in patients with no CR (mean: 1.02, 95\% conf. interval 0.89-1.14, median value $0.87, \mathrm{p}=0.031$, U Mann-Whitney test, Figure 2C). Fold-change $\mathrm{CR} /$ no $\mathrm{CR}$ for average values was 0.69 , while for median values 0.89 .

When the similar analysis was carried out for HSP90AB1, a trend was noted for lower expression in patients with CR (mean: 0.66, 95\% conf. interval 0.48-0.83, median value 0.55 ) than in patients with no CR (mean: $0.80,95 \%$ conf. interval $0.70-0.89$, median value $0.74, \mathrm{p}=0.054$, U Mann-Whitney test, Figure 2D). Fold change $\mathrm{CR} /$ no $\mathrm{CR}$ for average values was 0.82 , while for median values 0.74 . 


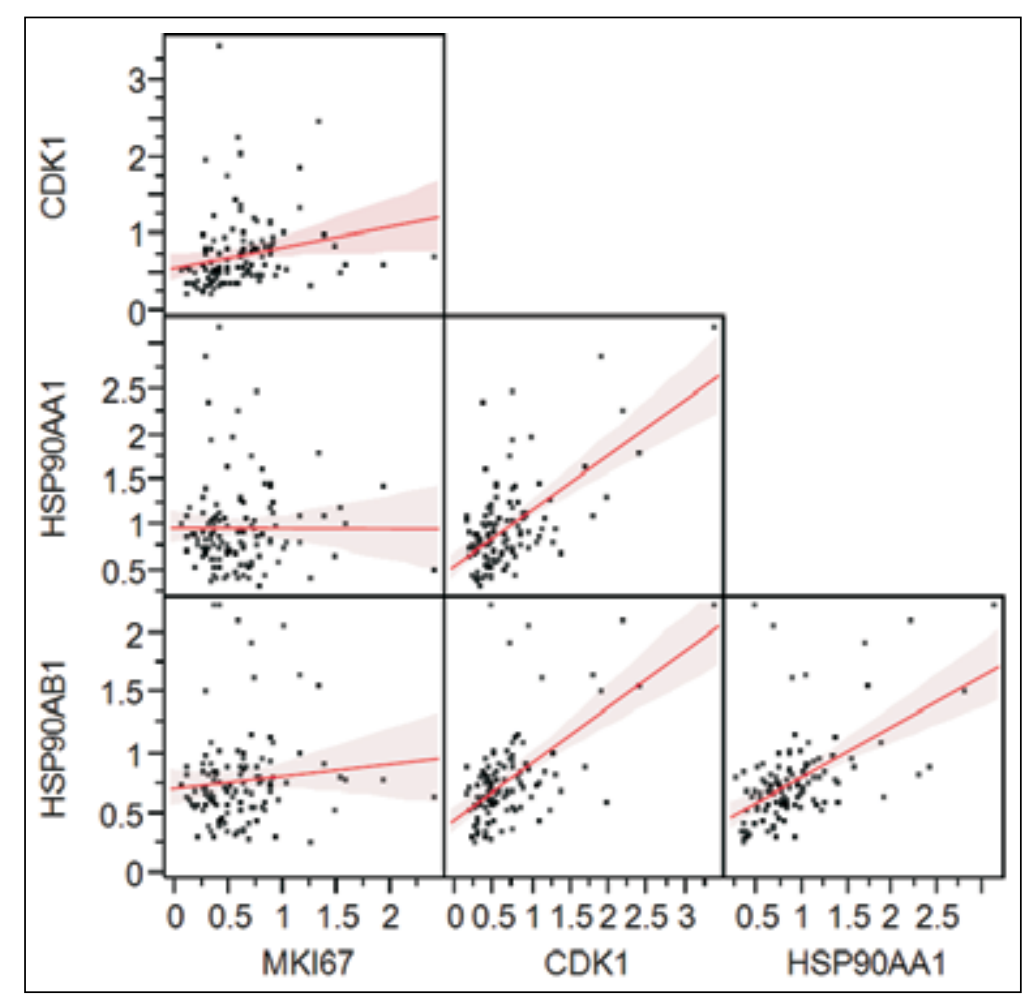

Figure 1. Pairwise correlation plots for all 4 analyzed transcripts (MKI67, CDK1, HSP90AA1, HSP90AB1). On both axes gene expression values are shown, with points representing individual patients. Regression lines are fitted, with $95 \%$ confidence intervals (shaded). Much stronger correlations were observed between CDK1 and both HSP90 transcripts than between these three genes and MKI67.

\section{Ratios of proliferation and HSP90 genes}

We calculated the ratios of both proliferation genes analyzed (MKI67 and CDK1) to each of HSP90 transcripts (AA1 and AB1), in total four pairwise combinations. All four calculated ratios showed significant differences between $\mathrm{CR}$ and no $\mathrm{CR}$ groups, more pronounced when HSP90AA1 was involved ( $p=0.002$ and $p=0.005$ for CDK1 and MKI67 genes, respectively), while slightly less prominent for HSP90AB1 $(\mathrm{p}=0.054$ and $\mathrm{p}=0.018$ for CDK1 and MKI67, resp.) The most significant predictor of $\mathrm{pCR}$ was the ratio of CDK1 transcript to HSP90AA1 transcript. CDK1/HSP90AA1 ratio was significantly higher in CR group (mean 1.06, median 0.99) than in no CR group (mean 0.73, median 0.68, see Figure 3, $\mathrm{p}=0.0023$, U Mann-Whitney test). Fold change of average ratio values between $\mathrm{CR}$ and no $\mathrm{CR}$ was 1.45 , very similar fold-change was observed for median values -1.46 .

\section{Diagnostic utility}

As the ratio of CDK1/HSP90AA1 showed significant differences between CR and no CR patients, we assessed whether it could be used as a diagnostic test, by calculating the area under the receiver operating characteristic (ROC) curve. The area was 0.72 confirming the diagnostic utility of the CDK1/HSP90AA1 ratio (see Figure 4).

\section{Discussion}

The data obtained in our study suggest that the sensitivity to chemotherapy in breast cancer is related more to the relationship between proliferation intensity (the higher, the more chemosensitive) and the tumor stress response (the higher, the more chemoresistant) than to each of the parameters alone. Only when both parameters were considered as a ratio, the differences between the tumors with pathological complete response and the more chemoresistant cancers were statistically significant. Ratio of CDK1 to HSP90AA1 gene expression was better predictive of response to chemotherapy than proliferation rate measured by Ki67 transcript (MKI67). In fact, the majority of observed discriminative power came from HSP90AA1/ /AB1 transcripts expression, not from MKI67/CDK1 genes.

One of the first studies to correlate heat shock proteins expression with chemosensitivity was the 

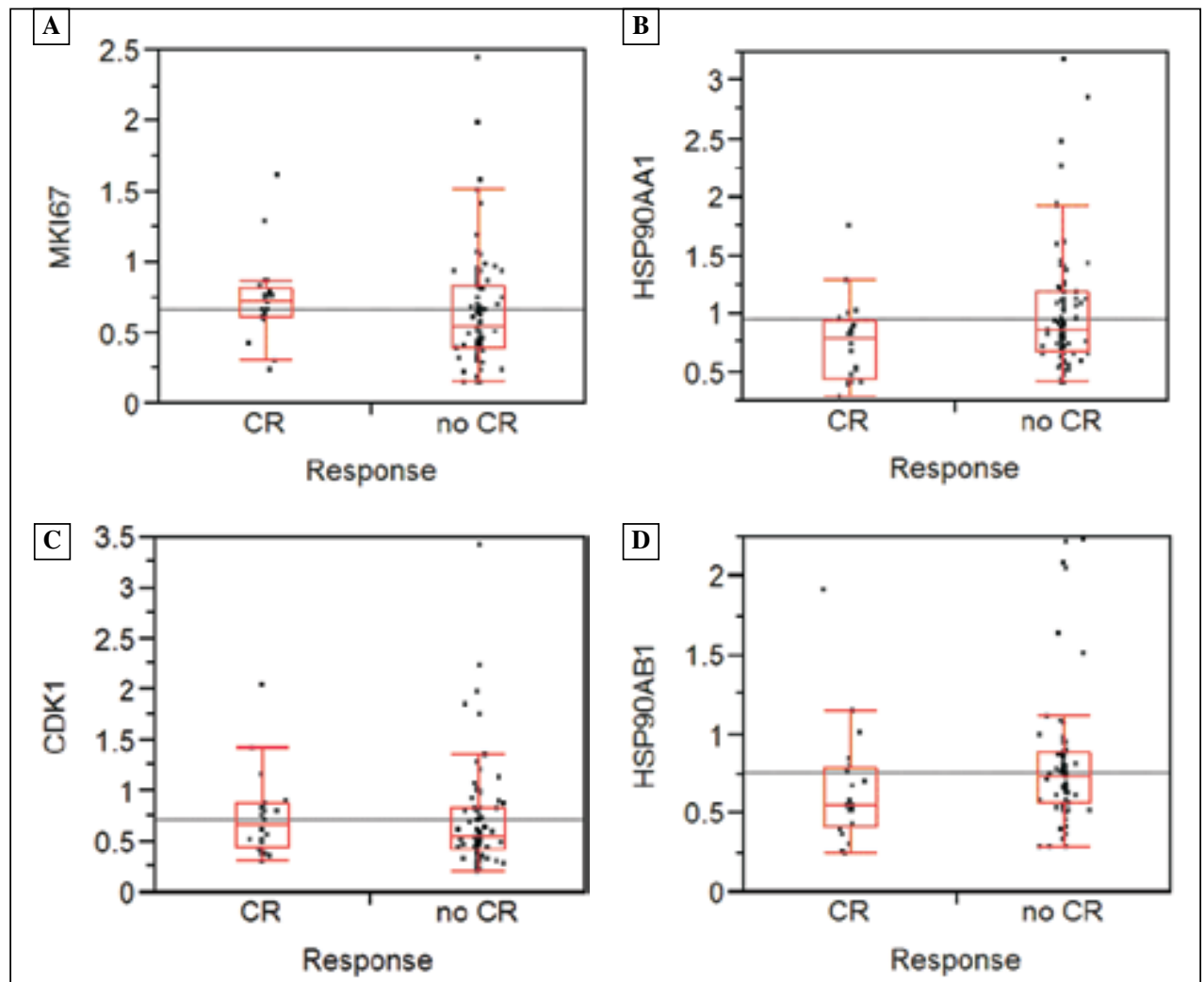

Figure 2. Differences in gene expression in analyzed transcripts between patients with pathological complete response $(\mathrm{CR})$ and no complete response (no CR). Box-and-whisker plots are shown in both groups. Gene expression values were compared by U Mann-Whitney test. For MKI67 and CDK1 transcripts the differences are not significant, $\mathrm{p}=0.099$ and $p=0.35$, respectively. Difference in gene expression of HSP90AA1 significantly differentiated both groups $(p=0.031)$, while for HSP90AB1 it was of borderline significance $(p=0.054)$.

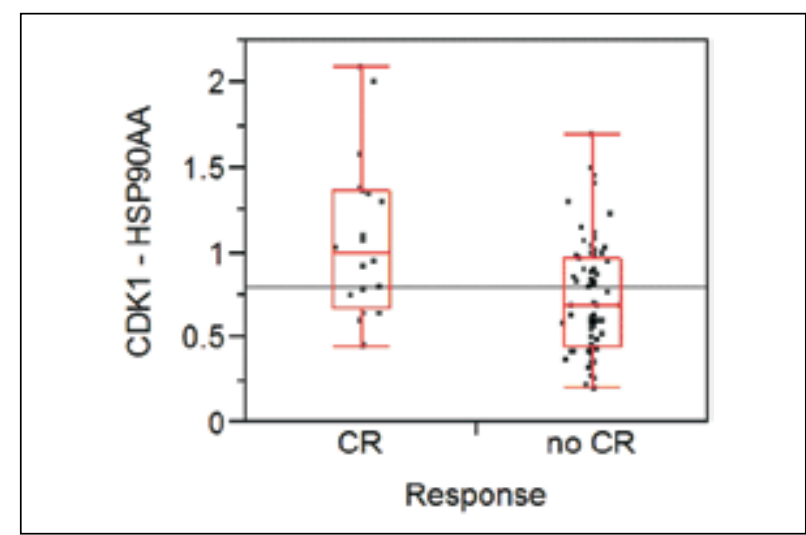

Figure 3. Relative expression of CDK1 to HSP90AA1 gene (measured by the ratio of both gene expression values) between patients in CR and no CR groups. The difference is statistically significant, $\mathrm{p}=0.0023$, U Mann-Whitney test.

analysis by Vargas-Roig et al. [4]. Authors analyzed the levels of Hsp70 and Hsp27 proteins and observed some association of high expression with tumor chemoresistance, although they were unable to identify any relation with immunohistochemically assessed proliferation rate.

HSP90 expression was first analyzed in breast cancer in 1999 [5], but until 2007 there was no systematic approach to associate it with clinical parameters. The negative impact of high HSP90 on patient survival was demonstrated by Pick et al. [6] by analysis of cell lines and patient-derived material in tissue microarray samples. High HSP90 protein staining was associated with shorter disease-free survival time, mainly in patients with early (node-negative) breast cancer. HSP90 was associated with disease-free survival time also when considered in the context of tumor size, steroid receptors and HER2 expression. High HSP90 expression was strongly associated with high nuclear grade, positive HER2 staining, larger tumor size, steroid receptors and lymph node metastases.

Significant acceleration in the understanding of HSP90 role in breast cancer has been achieved by analysis of multiple microarray datasets. This has been addressed by Cheng et al. [7] by compiling of more 


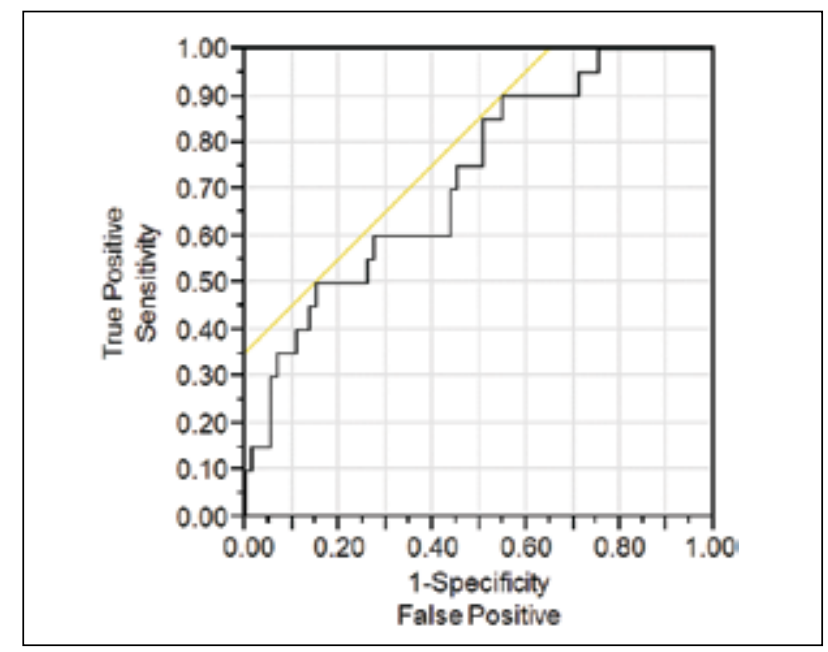

Figure 4. Diagnostic utility of CDK1/HSP90AA1 ratio to differentiate between $\mathrm{CR}$ and no CR patients, assessed by receiver operating characteristic (ROC) curve. The area under ROC curve was 0.72 , confirming the diagnostic utility of the CDK1/HSP90AA1.

than 4 thousand of samples from more than 20 datasets. The authors found that HSP90AA1 was associated with risk of death in triple-negative breast cancer, while HSP90AB1 was highly associated with risk of death in hormone-receptor positive breast cancer. Both genes were significantly associated with disease-free and overall survival in the whole cohort of patients.

Authors used also The Cancer Genome Atlas data [8] to demonstrate that the increased expression of each of HSP90 transcripts (cytoplasmic isoforms HSP90AA1 and AB1) might be driven either by amplifications of these genes or amplification of HSF1 transcription factor gene. When authors considered all reasons of activation of HSP90 gene (including HSF1 amplification) and analyzed its association with survival in all samples from 23 datasets gathered in the study, this upregulation was associated with the risk of death, especially in estrogen-receptor positive cancer. The largest effect was seen on the risk of recurrence in triple negative breast cancer.

Taking into account the potentially increased aggressiveness, related to high HSP expression, the use of HSP inhibition seem to be the rational option of novel anti-cancer therapy. This concept has been evaluated in many cancers [9-16], including numerous attempts in pre-clinical and clinical breast cancer models [17-27] (reviewed in [28]).

However, HSP is probably not acting alone in determination of breast cancer aggressiveness. Song et al. [29] analyzed by immunohistochemistry the effect of HSP90 expression on breast cancer relapse risk and found that increased HSP90 expression is significantly associated with its shortening. They noted that this effect is particularly pronounced in cases with PTEN loss or PI3K overexpression and that this interaction is clear in HER2 positive cases. Nevertheless, also in triple negative patients or luminal B subtype there was also a trend for worse prognosis related to higher HSP90 expression. TOPO2-HSP90 complex has also been indicated by Barker $\mathrm{et} \mathrm{al}$. as a potential valuable chemotherapeutic target [30].

Obviously, not only HSP90 was studied in the context of chemoresponsiveness. Important observations indicating that other heat shock proteins also play a role were published for HSP70 [31] and HSP27 [32-34]. The association of HSP90 gene expression with different pathological and clinical features of breast cancer has not yet been studied extensively in the novel setting of molecular subtypes of this disease. However, additionally to the suggestions that the role may be particularly associated with HER2-positive disease, there were also observations that HSP90 exhibits low expression in the lobular histotype [35].

In summary, HSP90 has been linked to chemoresistance in different clinical settings including breast cancer [36-41].The described study of Song et al. [29] corroborates the observations that low HSP90 expression is a positive prognostic feature. In our study we pinpointed the predictive aspect of the association of HSP90 with proliferation markers and its association with the higher response to chemotherapy. HSP90AA1 and AB1 genes exhibit low expression in breast cancers highly sensitive to chemotherapy and may indicate the patients with higher probability of pathological complete response. High HSP90 gene expression may be one of the mechanisms driving chemoresistance or might be a part of tumor aggressive phenotype. The ratio of HSP90AA1 to proliferation-related markers (CDK1 or MKI67) may be even better predictor of pCR chance, with higher expression of proliferation genes and lower stress response in patients sensitive to chemotherapy.

\section{Acknowledgements}

This work was supported by grant of Polish National Science Center N N402 686140 to MJ (molecular and data analysis). The clinical part of the project was partially supported by Polish National Center of Research and Development MILESTONE project - Molecular diagnostics and imaging in individualized therapy for breast, thyroid and prostate cancer, grant no. STRATEGMED2/267398/4/NCBR/2015. We thank Mrs. Aneta Kus, Monika Momot and Agnieszka Felisiak for excellent secretarial assistance and Mrs. Renata Cyplinska for her skillful technical lab support. 


\section{References}

1. Nishimura R, Osako T, Okumura Y, Hayashi M, Arima N. Clinical significance of Ki-67 in neoadjuvant chemotherapy for primary breast cancer as a predictor for chemosensitivity and for prognosis. Breast Cancer. 2010;17:269-275. doi: 10.1007/s12282-009-0161-5.

2. Bria E, Carbognin L, Furlanetto J et al. Impact of neoadjuvant single or dual HER2 inhibition and chemotherapy backbone upon pathological complete response in operable and locally advanced breast cancer: Sensitivity analysis of randomized trials. Cancer Treat Rev. 2014;40:847-856. doi: 10.1016/j.ctrv.2014.05.001.

3. Tyszkiewicz T, Jarzab M, Szymczyk C et al. Epidermal differentiation complex (locus 1q21) gene expression in head and neck cancer and normal mucosa. Folia Histochem Cytobiol. 2014;52:79-89. doi: 10.5603/FHC.2014.0018.

4. Vargas-Roig LM, Gago FE, Tello O, Aznar JC, Ciocca DR. Heat shock protein expression and drug resistance in breast cancer patients treated with induction chemotherapy. Int J Cancer. 1998;79:468-475. doi: 10.1002/(SICI)10970215(19981023)79:5<468::AID-IJC4>3.0.CO;2-Z.

5. Yano M, Naito Z, Yokoyama $M$ et al. Expression of hsp90 and cyclin D1 in human breast cancer. Cancer Lett. 1999;137:45-51. PMID: 10376793.

6. Pick E, Kluger Y, Giltnane JM et al. High HSP90 expression is associated with decreased survival in breast cancer. Cancer Res. 2007;67:2932-2937. doi: 10.1158/0008-5472. CAN-06-4511.

7. Cheng Q, Chang JT, Geradts J et al. Amplification and high -level expression of heat shock protein 90 marks aggressive phenotypes of human epidermal growth factor receptor 2 negative breast cancer. Breast Cancer Res. 2012;14:R62. doi: $10.1186 /$ bcr 3168 .

8. Comprehensive molecular portraits of human breast tumours. Nature. 2012;490:61-70. doi: 10.1038/nature11412.

9. Banerji U, Judson I, Workman P. The clinical applications of heat shock protein inhibitors in cancer - present and future. Curr Cancer Drug Targets. 2003;3:385-390. PMID: 14529390.

10. Acquaviva J, He S, Sang J et al. mTOR inhibition potentiates HSP90 inhibitor activity via cessation of HSP synthesis. Mol Cancer Res. 2014;12:703-713. doi: 10.1158/1541-7786. MCR-13-0605.

11. Garcia-Carbonero R, Carnero A, Paz-Ares L. Inhibition of HSP90 molecular chaperones: moving into the clinic. Lancet Oncol. 2013;14:e358-e369. doi: 10.1016/S14702045(13)70169-4.

12. Jego G, Hazoumé A, Seigneuric R, Garrido C. Targeting heat shock proteins in cancer. Cancer Lett. 2013;332:275-285. doi: 10.1016/j.canlet.2010.10.014.

13. Jhaveri K, Modi S. HSP90 inhibitors for cancer therapy and overcoming drug resistance. Adv Pharmacol. 2012;65:471-517. doi: 10.1016/B978-0-12-397927-8.00015-4.

14. Lu X, Xiao L, Wang L, Ruden DM. Hsp90 inhibitors and drug resistance in cancer: the potential benefits of combination therapies of Hsp90 inhibitors and other anti-cancer drugs. Biochem Pharmacol. 2012;83:995-1004. doi: 10.1016/j. bcp.2011.11.011.

15. Neckers L, Workman P. Hsp90 molecular chaperone inhibitors: are we there yet? Clin Cancer Res. 2012;18:64-76. doi: 10.1158/1078-0432.CCR-11-1000.

16. Scaltriti M, Dawood S, Cortes J. Molecular pathways: targeting Hsp90 - who benefits and who does not. Clin Cancer Res. 2012;18:4508-4513. doi: 10.1158/1078-0432. CCR-11-2138.
17. Barrott JJ, Hughes PF, Osada T et al. Optical and radioiodinated tethered Hsp90 inhibitors reveal selective internalization of ectopic Hsp90 in malignant breast tumor cells. Chem Biol. 2013;20:1187-1197. doi: 10.1016/j.chembiol.2013.08.004.

18. Beliakoff J, Whitesell L. Hsp90: an emerging target for breast cancer therapy. Anticancer Drugs. 2004;15:651-662. doi: 10.1097/01.cad.0000136876.11928.be.

19. Beliakoff J, Bagatell R, Paine-Murrieta G, Taylor CW, Lykkesfeldt AE, Whitesell L. Hormone-refractory breast cancer remains sensitive to the antitumor activity of heat shock protein 90 inhibitors. Clin Cancer Res. 2003;9:4961-4971. PMID: 14581371.

20. Chandarlapaty S, Scaltriti M, Angelini P et al. Inhibitors of HSP90 block p95-HER2 signaling in trastuzumab-resistant tumors and suppress their growth. Oncogene. 2010;29:325-334. doi: 10.1038/onc.2009.337.

21. Haroon ZA, Amin K, Saito W, Wilson W, Greenberg CS, Dewhirst MW. SU5416 delays wound healing through inhibition of TGF- $\beta$ activation. Cancer Biol Ther. 2002;1:121-126. doi: 10.4161/cbt.55.

22. de Candia P, Solit DB, Giri D et al. Angiogenesis impairment in Id-deficient mice cooperates with an Hsp90 inhibitor to completely suppress HER2/neu-dependent breast tumors. Proc Natl Acad Sci USA . 2003;100:12337-12342. doi: 10.1073/ pnas.2031337100.

23. De Mattos-Arruda L, Cortes J. Breast cancer and HSP90 inhibitors: is there a role beyond the HER2-positive subtype? Breast. 2012;21:604-607. doi: 10.1016/j.breast.2012.04.002.

24. Gee JMW. Chaperone-mediated destruction of erbB2: relevance to tyrosine kinase inhibitors. Breast Cancer Res. 2002;4:205-206. doi: 10.1186/bcr546.

25. Jensen MR, Schoepfer J, Radimerski T et al. NVP-AUY922: a small molecule HSP90 inhibitor with potent antitumor activity in preclinical breast cancer models. Breast Cancer Res. 2008;10:R33. doi: 10.1186/bcr1996.

26. Jhaveri K, Chandarlapaty S, Lake D et al. A phase II open -label study of ganetespib, a novel heat shock protein 90 inhibitor for patients with metastatic breast cancer. Clin Breast Cancer. 2014;14:154-160. doi: 10.1016/j.clbc.2013.12.012.

27. Kim LS, Kim JH. Heat shock protein as molecular targets for breast cancer therapeutics. J Breast Cancer. 2011;14:167-174. doi: $10.4048 / \mathrm{jbc}$.2011.14.3.167.

28. Zagouri F, Sergentanis TN, Chrysikos D, Papadimitriou CA, Dimopoulos M-A, Psaltopoulou T. Hsp90 inhibitors in breast cancer: a systematic review. Breast. 2013;22:569-578. doi: 10.1016/j.breast.2013.06.003.

29. Song CH, Park SY, Eom K-Y et al. Potential prognostic value of heat-shock protein 90 in the presence of phosphatidylinositol-3-kinase overexpression or loss of PTEN, in invasive breast cancers. Breast Cancer Res. 2010;12:R20. doi: 10.1186/bcr2557.

30. Barker CR, Hamlett J, Pennington SR et al. The topoisomerase II-Hsp90 complex: a new chemotherapeutic target? Int J Cancer. 2006;118:2685-2693. doi: 10.1002/ijc.21717.

31. Hyun JJ, Lee HS, Keum B et al. Expression of heat shock protein 70 modulates the chemoresponsiveness of pancreatic cancer. Gut Liver. 2013;7:739-746. doi: 10.5009/ gnl.2013.7.6.739.

32. Grzegrzółka J, Kurnol K, Piotrów P et al. Hsp-27 expression in invasive ductal breast carcinoma. Folia Histochem Cytobiol. 2012;50:527-533. doi: 10.5603/16717.

33. Lee CH, Hong HM, Chang YY, Chang WW. Inhibition of heat shock protein (Hsp) 27 potentiates the suppressive 
effect of Hsp90 inhibitors in targeting breast cancer stem -like cells. Biochimie. 2012;94:1382-1389. doi: 10.1016/j. biochi.2012.02.034.

34. McConnell JR, McAlpine SR. Heat shock proteins 27, 40, and 70 as combinational and dual therapeutic cancer targets. Bioorganic Med Chem Lett. 2013;23:1923-1928. doi: 10.1016/j. bmcl.2013.02.014.

35. Zagouri F, Sergentanis T, Nonni A et al. Decreased Hsp90 expression in infiltrative lobular carcinoma: an immunohistochemical study. BMC Cancer. 2010;10:409. doi: 10.1186/14712407-10-409.

36. Chu S-H, Liu Y-W, Zhang L, Liu B, Li L, Shi J-Z. Regulation of survival and chemoresistance by HSP90AA1 in ovarian cancer SKOV3 cells. Mol Biol Rep. 2013;40:1-6. doi: 10.1007/ s11033-012-1930-3.

37. Collura A, Lagrange A, Svrcek M et al. Patients with colorectal tumors with microsatellite instability and large deletions in HSP110 T17 have improved response to 5-fluorouracil-based chemotherapy. Gastroenterology. 2014;146:401-411.e1. doi: 10.1053/j.gastro.2013.10.054.

38. Guttmann DM, Koumenis C. The heat shock proteins as targets for radiosensitization and chemosensitization in cancer. Cancer Biol Ther. 2011;12:1023-1031. doi: 10.4161/cbt.12.12.18374.

39. Simpson NE, Lambert WM, Watkins R et al. High levels of Hsp90 cochaperone $\mathrm{p} 23$ promote tumor progression and poor prognosis in breast cancer by increasing lymph node metastases and drug resistance. Cancer Res. 2010;70:8446-8456. doi: 10.1158/0008-5472.CAN-10-1590.

40. Solit DB, Basso AD, Olshen AB, Scher HI, Rosen N. Inhibition of heat shock protein 90 function down-regulates Akt kinase and sensitizes tumors to Taxol. Cancer Res. 2003;63:2139-2144. PMID: 12727831.

41. Stope MB, Weiss M, Preuss M et al. Immediate and transient phosphorylation of the heat shock protein 27 initiates chemoresistance in prostate cancer cells. Oncol Rep. 2014;32:2380-2386. doi: 10.3892/or.2014.3492.

Submitted: 17 July, 2016 Accepted after reviews: 28 December, 2016 Available as AoP: 3 January, 2017 